\title{
A Hybrid Range-Free Localization Scheme in Wireless Sensor Networks
}

\author{
Jianbo $\mathrm{Li}^{1,2}$ \\ jbli@mail.ustc.edu.cn \\ ${ }^{1}$ Department of Computer Science and Technology \\ University of Science and Technology of China \\ Hefei, 230027, Anhui Province, P.R. China
}

Work in Progress

\begin{abstract}
The localization information of each node in Wireless Sensor Networks (WSNs) is of vital importance to most location-aware applications. In this paper, a hybrid range-free localization scheme is proposed by adopting DV-Hop and weighted Centroid localization schemes. The nodes in the sensor network are firstly grouped into clusters, and then Weighted Centroid localization scheme is employed to achieve the location of most sensor nodes in each cluster. Finally, location of "undetermined" nodes is computed by using DV-Hop. The proposed localization algorithm achieves a balance between cost and accuracy, while yielding an acceptable accuracy and obtaining the scalability of sensor networks. Simulation results show that our proposed algorithm performs well under the condition that comparatively high density of anchor nodes are deployed.
\end{abstract}

\section{Categories and Subject Descriptors}

C.2.1 [Computer Communication Networks]: Network Architecture and Design -Distributed Networks, Wireless Communication

\section{General Terms}

Algorithm, Design, Performance

\section{Keywords}

Localization, weighted centroid, DV-Hop, sensor networks

\section{INTRODUCTION}

Location information plays a crucial role in understanding the application context in WSNs][3][6]. Localization schemes for wireless sensor networks can be classified as range-based or range-free ones. They differ in the information used for localization. Range-based localization schemes usually deduce the distances based on the received signal strength measurement or

Permission to make digital or hard copies of all or part of this work for personal or classroom use is granted without fee provided that copies are not made or distributed for profit or commercial advantage and that copies bear this notice and the full citation on the first page. To copy otherwise, or republish, to post on servers or to redistribute to lists, requires prior specific permission and/or a fee.

INFOSCALE 2007, June 6-8, Suzhou, China

Copyright () 2007 ICST 978-1-59593-757-5

DOI 10.4108/infoscale.2007.221

\author{
Mingjun Xiao ${ }^{1}$ \\ Hongli $\mathrm{Xu}{ }^{1}$ \\ xiaomj@ustc.edu.cn hlxu3@mail.ustc.edu.cn \\ ${ }^{2}$ Information engineering college \\ Qingdao university \\ Qingdao, 266071, Shandong Province ,P.R.China
}

time-of-flight of communication signal from a sender to a receiver. In contrast, range-free localization schemes never need the absolute point-to-point distances or angles to estimate the node's location, rendering it more appealing compared with range-based ones.

This paper proposes a hybrid range-free localization consisting of three phases: cluster and outer anchors construction, Weighted Centroid[4] localizing "determined" nodes, and DV-Hop [2]localizing "undetermined" nodes. The "determined" nodes mean the nodes whose location can be computed, while the "undetermined" nodes are opposite. The main contribution of this paper embodies two aspects: one is the integration of Weighted Centroid and DVHop to achieve a trade-off between accuracy and cost. The other is that we propose a novel method to select outer anchors evenly distributed in deployment regions to alleviate the "large-scale estimation error" problem in Centroid scheme.

\section{OUR LOCALIZATION SCHEME}

\subsection{Cluster and Outer Anchors Construction}

The objective of this phase is to form clusters and select cluster heads (CHs) by using a method analogue with GAF[5]. Initially the sensing filed was divided into virtual grids with an edge of $\mathrm{r}$ units, based on the position and radio range of anchor nodes, such that all anchors in grid A can communicate with all anchor nodes in A's neighboring grid B. In each cluster, the anchor node with the biggest ID was chosen to be cluster head and other anchor nodes plus regular sensor nodes became cluster members. When the number of anchors in a cluster is less than 8 , the nodes in that cluster are simply classified into "undetermined" nodes to alleviate the location error.

Next, we propose algorithm slightly different from [1] to choose $\beta$ outer anchor nodes in each cluster by using a vector $\mathrm{V}$.

1. initialize a vectors $V$ of size $\beta$ to be empty to hold outer anchors.

2. Select an anchor node $\boldsymbol{a}_{0}$ with the smallest ID and then select the anchor node $\boldsymbol{a}_{1}$ to maximize $\boldsymbol{d}_{0,1} . V[1] \leftarrow \boldsymbol{a}_{1}$.

3. Select anchor node $\boldsymbol{a}_{2}$ to maximize $\boldsymbol{d}_{1,2} . V[2] \leftarrow \boldsymbol{a}_{2}$.

4. Select anchor node $\boldsymbol{a}_{3}$ to maximize $\boldsymbol{d}_{2,3}+\boldsymbol{d}_{1,3} . V[3] \leftarrow \boldsymbol{a}_{3}$.

5. Select anchor node $\boldsymbol{a}_{4}$ to maximize $\boldsymbol{d}_{3,4} . V[4] \leftarrow \boldsymbol{a}_{4}$.

6. $j \leftarrow 4$

7. while $j \neq \beta$ for $i \leftarrow 1$ to $j$ 
select anchor node $\boldsymbol{a}_{\boldsymbol{k}} \notin V$ such that $\left(\boldsymbol{d}_{\boldsymbol{i}, \boldsymbol{k}}+\boldsymbol{d}_{(\boldsymbol{i}+1) \% j, \boldsymbol{k}}\right)$ is maximized and $\left(\boldsymbol{d}_{\boldsymbol{i}, \boldsymbol{k}}-\boldsymbol{d}_{(\boldsymbol{i}+\mathbf{1}) \% \boldsymbol{j}, \boldsymbol{k}}\right)$ is minimized.

$V[j+i] \leftarrow a_{k}$

$j \leftarrow 2 j$

return $\boldsymbol{V}$

As shown in Fig.1 under random node placement, $\beta$ outer anchors were selected to approximately enclose the whole area, where $\beta=8$.

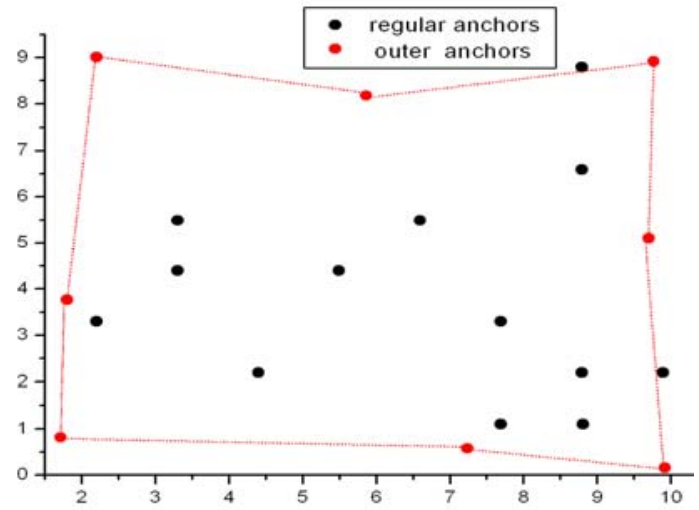

Figure 1.An example of outer anchors.

\subsection{Using Weighted Centroid Scheme}

To alleviate the influence of location error, we adopt the Weighted Centroid method proposed by [4] to calibrate location error. Now, the new node location estimation can be expressed in the following formula:

$$
\left(X_{e s t}, Y_{e s t}\right)=\left(\frac{\sqrt[\alpha]{P_{1}} X_{1}+\ldots+\sqrt[\alpha]{P_{n}} X_{n}}{\sqrt[\alpha]{P_{1}}+\ldots+\sqrt[\alpha]{P_{n}}}, \frac{\sqrt[\alpha]{P_{1}} Y_{1}+\ldots+\sqrt[\alpha]{P_{n}} Y_{n}}{\sqrt[\alpha]{P_{1}}+\ldots+\sqrt[\alpha]{P_{n}}}\right)
$$

Where $\alpha$ is called the path loss exponent, $P_{i}$ is the average RSSI metric for outer anchors (in $\mathrm{mW}$ ) determined in phase one.

\subsection{Using DV-Hop Scheme}

In this phase, a slightly different DV-Hop scheme [2] version is employed to solve the "undetermined" nodes problem. In this work, each outer anchor node $A_{i}$ produced in phase one initiates a flood of the network by broadcasting a packet containing its position, ID, and a counter initialized to one, thus reducing the number of nodes participating the flood progress which helps to save much energy. Once an "undetermined" node can calculate the distance estimation to more than 3 outer anchors in the plane, it uses triangulation (Multilateration) to estimate its location.

\section{PRELIMINARY RESULTS}

We used a c++ event tool to simulate 100 nodes randomly deployed within $10 \mathrm{R} \times 10 \mathrm{R}$ area so as to compare the performance of the range-free localization algorithms among ours, Centroid, and DV-Hop. The location error was used as the metric and other parameters were set as follows: $\alpha=2, \beta=8$. Being restrict to the space, only the location error under the varying node densities, with anchors heard set 8 and anchor to node range ratio set 5 ,are given as shown in Figure 2

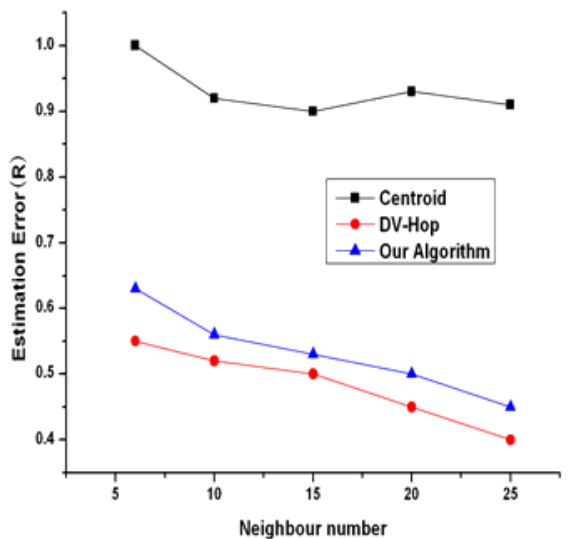

Figure 2. Estimation error under varying ND.

\section{Future Works}

The proposed method is an inherently coarse-grained localization scheme, which directs us to make efforts to achieve much finer precision in the future work .Furthermore, another metric to measure the cost of range-free localization schemes should be developed to verify the proposed algorithm is less cost intensive.

\section{ACKNOWLEDGMENTS}

This work thanks the support by the National Grand Fundamental Research 973 Program of China under Grant No.2006CB303000.

\section{REFERENCES}

[1] A. Ahmed, H. Shi, and Y. Shang, "Sharp: A new approach to relative localization in wireless sensor network," 25th IEEE International Conference on Distributed Computing Systems Workshop, ICDCSW, pp. 892-898, 200

[2] D. Niculescu and B. Nath, "Ad hoc positioning system (APS)," GLOBECOM'01. IEEE Global Telecommunications Conference 5, pp. 2926-2931, 2001.

[3] S. Meguerdichian, F. Koushanfar, M. Potkonjak, and M.B. Srivastava,"Coverage Problems in Wireless Ad-hoc Sensor Neworks," Proceedings of IEEE Infocom 2001, Ankorange, Alaska, April 2001.

[4] Xingfa Shen, Zhi Wang, Ruizhong Lin et.al, Connectivity and RSSI Based Localization Scheme for Wireless Sensor Networks, ICIC 2005, Part II, LNCS 3645, pp. 578-587, 2005.

[5] Xu Y, Heidemann J, Estrin D. Geography-informed energy conserva-tion for ad hoc routing In: Proc 7th Annual Int'l Conf on Mobile Computing and Networking (MobiCom), Rome, Italy, July 2001. 70-84.

[6] Y.B. Ko and N. H. Vaidya, "Location-Aided Routing (LAR) Mobile Ad Hoc Networks," Proceedings of the fourth annual international conference on Mobile computing and networking (MobiCom 98), Dallas, Texas, October 1998, pp. 66-75. 